\title{
A Mathematical Analysis of a Model Involving an Integrable Equation for Wave Packet Envelope
}

\author{
Melike Kaplan (i) ${ }^{1}$ and Arzu Akbulut $\mathbb{D}^{2}$ \\ ${ }^{1}$ Kastamonu University, Faculty of Engineering and Architecture, Department of Computer Engineering, Kastamonu, Turkey \\ ${ }^{2}$ Eskişehir Osmangazi University, Art-Science Faculty, Department of Mathematics-Computer, Eskisehir, Turkey \\ Correspondence should be addressed to Melike Kaplan; mkaplan@kastamonu.edu.tr
}

Received 23 November 2021; Revised 17 December 2021; Accepted 6 January 2022; Published 9 February 2022

Academic Editor: Barbara Martinucci

Copyright (C) 2022 Melike Kaplan and Arzu Akbulut. This is an open access article distributed under the Creative Commons Attribution License, which permits unrestricted use, distribution, and reproduction in any medium, provided the original work is properly cited.

This study investigates new optical solutions to a model with an integrable equation for wave packet envelopes. For this purpose, we have employed two reliable techniques involving the modified extended tanh function and the exponential rational function procedures. We have also given the $3 \mathrm{D}$ graphics of the obtained solutions.

\section{Introduction}

Numerous complex phenomena that are encountered in relativity, fluid dynamics, mathematical physics, economics, and optical fibers are modeled via nonlinear partial differential equations (NPDEs) [1]. Recently, new methods have been recommended for finding solutions to the NPDEs. Some notable studies have been discussed. Alquran has obtained periodic solutions and bell-shaped soliton solutions to the two-component evolutionary system of homogeneous $\mathrm{KdV}$ equations of the second order, the Gardner equation, Benjamin-Bona-Mahony equation, and the Cassama-Holm equation via the sine-cosine method [2]. Akbulut et al. have employed the $\exp (-\varphi(\xi))$-expansion procedure to the ill-posed Boussinesq equation and Zakharov-Kuznetsov-Benjamin-Bona-Mahony equation to obtain trigonometric, hyperbolic, and rational functions for these equations [3]. In 2015, Kaplan et al. applied the auxiliary equation procedure to the nonlinear Zoomeron equation, equal width wave equation, and coupled Higgs equation [4]. Ali et al. discussed cubic optical solitons in a polarization-preserving fiber described via nonlinear Schrödinger equation (NLSE) via the new extended direct algebraic method [5]. Yao et al. used the improved generalized Riccati equation mapping procedure to get traveling wave solutions for the higher-order Sasa-Satsuma equation [6]. Durur et al. applied the subequation procedure to find exact solutions for the KdV6 equation [7]. In 2021, Mirzazadeh et al. have employed the generalized Riccati equation mapping method [8]. Ma et al. have employed the first integral, the exp $(-\varphi(\xi))$-expansion, and sine-Gordon expansion procedures to extract soliton solutions of the Hirota-Maccari system [9]. In 2016, Osman used the generalized unified method for the Zakharov-Kuznetsov equation [10]. Inc et al. utilized the functional variable procedure [11]. Leta et al. applied the bifurcation technique to the $(2+1)$-dimensional Bogoyavlenskii coupled system [12]. Rezazadeh et al. applied the extended rational sin-cos method to the chiral nonlinear Schrödinger equation [13].

In recent years, optical solitary waves, temporal and spatial solitons, have been discussed by many researchers theoretically and experimentally. For example, in 2018, Biswas et al. have founded optical solitons for the Lakshmanan-Porsezian-Daniel model via the modified simple equation procedure [14]. Manafian, in 2016, has founded optical soliton solutions for NLSE via the tan $(\phi(\xi) / 2)$-expansion procedure [15]. Kudryashov, in 2020, 
has proposed a procedure to find highly-dispersive optical solitons of NPDEs [16].

This paper studied

$$
\begin{aligned}
i q_{t} & +a q_{x x}+i b q_{x x x}+c q_{x x x x}+d|q|^{4} q+e|q|^{2} q \\
& +f\left(|q|^{2}\right)_{x x} q=0,
\end{aligned}
$$

where $a, b, c, d, e$, and $f$ are parameters, and $q(x, t)$ is a complex-valued function. It is known that this equation is the generalization of some famous equations. It is utilized to describe traveling wave solutions in optical fibers. This equation is reduced to NLSE when we choose $b=c=d=f=0$, and it models the slowly varying envelope dynamics of a weakly nonlinear quasimonochromatic wave packet in dispersive media [17]. Also, by setting $b=c=f=0$ in equation (1), the cubic-quintic NLS (cqNLS) equation is obtained which is used in the nonlinear optics $[18,19]$. cqNLS is used for the nuclear hydrodynamics with Skyrme forces [20], the optical pulse propagations in dielectric media of non-Kerr type [21], and description of the boson gas with two and three-body interactions [22]. For this reason, we can consider equation (1) as a generalization of the NLSE. It could be utilized for describing nonlinear processes in optics [23].

The paper consists of the following sections: we present the preliminary information in Section 2. Then, we give a description of utilized techniques, respectively, and The modified extended tanh-function procedure and the exponential rational function procedure in Section 2.2. The application of the given methods is given in Section 3. Finally, the conclusion of the whole research is given in Section 4.

\section{Preliminary Information}

In this section, we firstly introduce the preliminary information for reducing NPDE to an ordinary differential equation (ODE). For this purpose, we will consider the following NPDE:

$$
P\left(q, q_{t}, q_{x}, q_{t t}, q_{x x}, q_{x t}, \ldots\right)=0,
$$

where $P$ inherits $q$ and its partial derivatives, and $q$ is a complex-valued function.

Wave transformation is given by

$$
q(x, t)=u(\xi) e^{i(k x-v t)}, \xi=x-c_{0} t,
$$

where $v, k$, and $c_{0}$ are the constants. If we apply transformation (3) to equation (2) and equate the real and imaginary parts to zero, we obtain two equation systems. Then, we solve these systems to find conditions for parameters and use the values of the parameters. Therefore, we find an ODE, which will be integrated with respect to $\xi$ possible times, as follows:

$$
Q\left(u, u \prime, u^{\prime \prime}, u^{\prime \prime \prime}, \ldots\right)=0
$$

where the partial derivative is given with respect to $\xi$ [24].
The modified extended tanh function and the exponential rational function procedures are applicable to this reduced ODE.

2.1. The Modified Extended Tanh-Function Procedure. We will assume the exact solution of equation (4) by

$$
u(\xi)=\sum_{i=0}^{n} a_{i}(\phi(\xi))^{i}+\sum_{j=1}^{n} b_{j}(\phi(\xi))^{-j},
$$

to employ the modified extended tanh-function procedure. Here, $a_{i}(i=0,1, \ldots, n), b_{j}(j=1, \ldots, n)$ are constants, and they will be calculated; $a_{n}$ and $b_{n}$ cannot be zero at the same time. In equation (5), the term $n$ is called the balancing term. This term can be found balancing the nonlinear terms and the highest order derivatives. $\phi(\xi)$ is the solution of following auxiliary equation:

$$
\phi^{\prime}(\xi)=\phi(\xi)^{2}+\beta
$$

where $\beta$ is given as a constant. The solutions of equation (6) are given in three cases as follows:

(1) When $\beta<0$, the hyperbolic solution is

$$
\phi(\xi)=-\sqrt{-\beta} \tanh (\sqrt{-\beta} \xi) \text { or } \phi(\xi)=-\sqrt{-\beta} \operatorname{coth}(\sqrt{-\beta} \xi) \text {. }
$$

(2) When $\beta>0$, the trigonometric solution is

$$
\phi(\xi)=\sqrt{\beta} \tan (\sqrt{\beta} \xi) \text { or } \phi(\xi)=\sqrt{-\beta} \cot (\sqrt{\beta} \xi) .
$$

(3) When $\beta=0$, the rational solution is

$$
\phi(\xi)=-\frac{1}{\xi}
$$

Surrogating equation (5) into equation (4) along with equation (6), then accepting all the coefficients of $\phi^{i}(\xi)$ is zero, we get an algebraic equation system for $k, v, c_{0}, a_{i}(i=$ $0, \ldots, n), b_{j}(j=1, \ldots, n)$. If we solve an obtained determining equation system with the Maple, we find a variety of exact solutions [25].

2.2. The Exponential Rational Function Procedure. We will assume the solution of equation (4) as follows [26, 27]:

$$
u(\xi)=\sum_{i=0}^{n} \frac{a_{i}}{\left(1+e^{\xi}\right)^{n}}
$$

where $a_{n}\left(a_{n} \neq 0\right)$ are constants. Here, $n$ is the balancing number as the previous technique. We surrogate equation (10) into equation (4) and collect all terms with the same order of $e^{i \xi}(i=0,1,2, \ldots)$ together, and we make into the left-hand side of equation (4) and another polynomial in $e^{i \xi}$. Then, we equate each coefficient of this polynomial to zero, and it gives a set of algebraic equations for $a_{n}$ unknown parameters. Finally, we solve the equation system to construct a variety of exact solutions for equation (4). 


\section{Application of the Given Methods}

In the current section, we will see new traveling wave solutions of equation (1). For this reason, firstly, we give the mathematical analysis of the considered equation.
3.1. Mathematical Analysis. If we substitute equation (3) into equation (1) and then equate the real and imaginary parts to zero, we find

$$
\begin{aligned}
& (b+4 c k) u_{\xi \xi \xi}+\left(2 a k-c_{0}-3 b k^{2}-4 c k^{3}\right) u_{\xi}=0, \\
& c u_{\xi \xi \xi \xi}+2 f u^{2} u_{\xi \xi}+2 f u_{\xi}^{2} u+\left(a-3 b k-6 c k^{2}\right) u_{\xi \xi}+d u^{5}+e u^{3}+\left(v-a k^{2}+b k^{3}+c k^{4}\right) u=0 .
\end{aligned}
$$

From the first equation in the above system, we obtain

$$
\begin{aligned}
b & =-4 c k, \\
c_{0} & =2 a k-3 b k^{2}-4 c k^{3} .
\end{aligned}
$$

Using (12) in the second equation in System (11), we get following reduced ODE:

$$
\begin{gathered}
c u_{\xi \xi \xi \xi}+2 f u^{2} u_{\xi \xi}+2 f u_{\xi}^{2} u+\left(a+6 c k^{2}\right) u_{\xi \xi} \\
+d u^{5}+e u^{3}+\left(v-a k^{2}-3 c k^{4}\right) u=0 .
\end{gathered}
$$

We will separately apply the modified extended tanhfunction procedure and the exponential rational function procedure to this reduced ODE to obtain different exact solutions.

3.2. Modified Extended Tanh-Function Procedure. Homogeneous balance is obtained as $n=1$ from equation (13); thus, the solution of equation (13) is given by

$$
u(\xi)=a_{0}+a_{1} \phi(\xi)+\frac{b_{1}}{\phi(\xi)} .
$$

Surrogating equation (14) along with equation (6) into equation (13), then assuming the coefficients of $\phi(\xi)^{j}$ as zero,

$$
\begin{aligned}
& d a_{1}^{5}+6 f a_{1}^{3}+24 c a_{1}=0, \\
& 5 d a_{0} a_{1}^{4}+10 f a_{0} a_{1}^{2}=0, \\
& 10 d a_{0}^{2} a_{1}^{3}+5 d a_{1}^{4} b_{1}+8 f a_{1}^{3} \beta+4 f a_{0}^{2} a_{1}+e a_{1}^{3}+6 f a_{1}^{2} b_{1}+12 a_{1} c k^{2}+40 c a_{1} \beta+2 a_{1} a=0, \\
& 10 d a_{0}^{3} a_{1}^{2}+20 d a_{0} a_{1}^{3} b_{1}+12 f a_{0} a_{1}^{2} \beta+3 e a_{0} a_{1}^{2}+4 f a_{0} a_{1} b_{1}=0, \\
& 5 d a_{0}^{4} a_{1}+30 d a_{0}^{2} a_{1}^{2} b_{1}+10 d a_{1}^{3} b_{1}^{2}+2 f a_{1}^{3} \beta^{2}-3 a_{1} c k^{4}+4 f a_{0}^{2} a_{1} \beta+8 f a_{1}^{2} b_{1} \beta \\
& \quad+12 a_{1} c k^{2} \beta-a_{1} a k^{2}+3 e a_{0}^{2} a_{1}+3 e a_{1}^{2} b_{1}+2 f a_{1} b_{1}^{2}+16 c a_{1} \beta^{2}+2 a_{1} a \beta+a_{1} v=0, \\
& b_{1}^{5} d+6 b_{1}^{3} \beta^{2} f+24 b_{1} \beta^{4} c=0, \\
& 5 a_{0} b_{1}^{4} d+10 a_{0} b_{1}^{2} \beta^{2} f=0, \\
& 10 a_{0}^{2} b_{1}^{3} d+4 a_{0}^{2} b_{1} \beta^{2} f+5 a_{1} b_{1}^{4} d+6 a_{1} b_{1}^{2} \beta^{2} f+12 b_{1} \beta^{2} c k^{2}+8 b_{1}^{3} \beta f+40 b_{1} \beta^{3} c 2 a b_{1} \beta^{2}+b_{1}^{3} e=0 .
\end{aligned}
$$

is acquired. If we solve System (15), we obtain values of the constants.

\subsubsection{Solution Family 1.}

$$
\begin{aligned}
& a_{0}=0, \\
& a_{1}=0, b_{1} \\
& a=a, d=-\frac{6 \beta^{2}\left(b_{1}^{2} f+4 c \beta^{2}\right)}{b_{1}^{4}},
\end{aligned}
$$

$$
\begin{aligned}
& e=-\frac{2 \beta\left(6 c \beta k^{2}+4 b_{1}^{2} f+20 c \beta^{2}+a \beta\right)}{b_{1}^{2}}, \\
& v=3 c k^{4}-12 c \beta k^{2}+a k^{2}-2 b_{1}^{2} f-16 c \beta^{2}-2 a \beta .
\end{aligned}
$$

When $\beta<0$,

$$
\begin{aligned}
q_{1}(x, t)= & \left(-\frac{b_{1}}{\sqrt{-\beta} \tanh \left(\sqrt{-\beta}\left(x-\left(8 c k^{3}+2 a k\right) t\right)\right)}\right) \\
& \times e^{i\left(k x-\left(3 c k^{4}-12 c \beta k^{2}+a k^{2}-2 b_{1}^{2} f-16 c \beta^{2}-2 a \beta\right) t\right)} .
\end{aligned}
$$


When $\beta>0$,

$$
\begin{aligned}
q_{2}(x, t)= & \left(\frac{b_{1}}{\sqrt{\beta} \tan \left(\sqrt{\beta}\left(x-\left(8 c k^{3}+2 a k\right) t\right)\right)}\right) \\
& \times e^{i\left(k x-\left(3 c k^{4}-12 c \beta k^{2}+a k^{2}-2 b_{1}^{2} f-16 c \beta^{2}-2 a \beta\right) t\right)} .
\end{aligned}
$$

When $\beta=0$,

$q_{3}(x, t)=\left(-b_{1}\left(x-\left(8 c k^{3}+2 a k\right) t\right)\right) e^{i\left(k x-\left(3 c k^{4}+a k^{2}-2 b_{1}^{2} f\right) t\right) .}$

\subsubsection{Solution Family 2.}

$$
\begin{aligned}
& a_{0}=0, \\
& a_{1}=a_{1}, \\
& b_{1}=0, \\
& a=a, \\
& d=-\frac{6\left(a_{1}^{2} f+4 c\right)}{a_{1}^{4}}, \\
& e=-\frac{2\left(6 c k^{2}+4 a_{1}^{2} \beta f+20 c \beta+a\right)}{a_{1}^{2}}, \\
& v=-2 a_{1}^{2} \beta^{2} f+3 c k^{4}-12 c \beta k^{2}+a k^{2}-16 c \beta^{2}-2 a \beta .
\end{aligned}
$$

When $\beta<0$,

$$
\begin{aligned}
q_{4}(x, t)= & \left(-a_{1} \sqrt{-\beta} \tanh \left(\sqrt{-\beta}\left(x-\left(8 c k^{3}+2 a k\right) t\right)\right)\right) \\
& \times e^{i\left(k x-\left(-2 a_{1}^{2} \beta^{2} f+3 c k^{4}-12 c \beta k^{2}+a k^{2}-16 c \beta^{2}-2 a \beta\right) t\right)} .
\end{aligned}
$$

When $\beta>0$,

$$
\begin{aligned}
q_{5}(x, t)= & \left(a_{1} \sqrt{\beta} \tan \left(\sqrt{\beta}\left(x-\left(8 c k^{3}+2 a k\right) t\right)\right)\right) \\
& \times e^{i\left(k x-\left(-2 a_{1}^{2} \beta^{2} f+3 c k^{4}-12 c \beta k^{2}+a k^{2}-16 c \beta^{2}-2 a \beta\right) t\right) .}
\end{aligned}
$$

When $\beta=0$,

$q_{6}(x, t)=\left(-\frac{a_{1}}{\left(x-\left(8 c k^{3}+2 a k\right) t\right)}\right) e^{i\left(k x-\left(3 c k^{4}+a k^{2}\right) t\right)}$.

\subsubsection{Solution Family 3.}

$$
\begin{aligned}
& a_{0}=0, \\
& a_{1}=a_{1}, \\
& b_{1}=a_{1} \beta, \\
& a=a,
\end{aligned}
$$

$$
\begin{aligned}
& d=-\frac{6\left(a_{1}^{2} f+4 c\right)}{a_{1}^{4}}, \\
& e=-\frac{2\left(6 c k^{2}-8 a_{1}^{2} \beta f-40 c \beta+a\right)}{a_{1}^{2}}, \\
& v=3 c k^{4}+24 c \beta k^{2}+a k^{2}-16 c \beta^{2}+4 a \beta .
\end{aligned}
$$

When $\beta<0$,

$$
\begin{aligned}
q_{7}(x, t)= & \left(\frac{a_{1} \sqrt{-\beta}\left(\tanh \left(\sqrt{-\beta}\left(\left(x-\left(8 c k^{3}+2 a k\right) t\right)\right)\right)^{2}-1\right)}{\tanh \left(\sqrt{-\beta}\left(\left(x-\left(8 c k^{3}+2 a k\right) t\right)\right)\right)}\right) \\
& \times e^{i\left(k x-\left(3 c k^{4}+24 c \beta k^{2}+a k^{2}-16 c \beta^{2}+4 a \beta\right) t\right)} .
\end{aligned}
$$

When $\beta>0$,

$$
\begin{aligned}
q_{8}(x, t)= & \left(\frac{a_{1} \sqrt{\beta}\left(\tan \left(\sqrt{\beta}\left(\left(x-\left(8 c k^{3}+2 a k\right) t\right)\right)\right)^{2}+1\right)}{\tanh \left(\sqrt{\beta}\left(\left(x-\left(8 c k^{3}+2 a k\right) t\right)\right)\right)}\right) \\
& \times e^{i\left(k x-\left(3 c k^{4}+24 c \beta k^{2}+a k^{2}-16 c \beta^{2}+4 a \beta\right) t\right)} .
\end{aligned}
$$

When $\beta=0$, we obtain the same solution as the third solution in solution family 2 .

\subsubsection{Solution Family 4.}

$$
\begin{aligned}
& a_{0}=0, \\
& a_{1}=a_{1}, \\
& b_{1}=-a_{1} \beta,
\end{aligned}
$$

$a=a$,

$$
d=-\frac{6\left(a_{1}^{2} f+4 c\right)}{a_{1}^{4}}
$$

$$
\begin{aligned}
& e=-\frac{2\left(6 c k^{2}-16 a_{1}^{2} \beta f-80 c \beta+a\right)}{a_{1}^{2}}, \\
& v=-32 a_{1}^{2} \beta^{2} f+3 c k^{4}-48 c \beta k^{2}+a k^{2}-256 c \beta^{2}-8 a \beta
\end{aligned}
$$

When $\beta<0$,

$$
\begin{aligned}
q_{9}(x, t)= & \left(\frac{a_{1} \sqrt{-\beta}\left(\tanh \left(\sqrt{-\beta}\left(\left(x-\left(8 c k^{3}+2 a k\right) t\right)\right)\right)^{2}+1\right)}{\tanh \left(\sqrt{-\beta}\left(\left(x-\left(8 c k^{3}+2 a k\right) t\right)\right)\right)}\right) \\
& \times e^{i\left(k x-\left(-32 a_{1}^{2} \beta^{2} f+3 c k^{4}-48 c \beta k^{2}+a k^{2}-256 c \beta^{2}-8 a \beta\right) t\right) .}
\end{aligned}
$$


When $\beta>0$,

$$
\begin{aligned}
q_{10}(x, t)= & \left(\frac{a_{1} \sqrt{\beta}\left(\tan \left(\sqrt{\beta}\left(\left(x-\left(8 c k^{3}+2 a k\right) t\right)\right)\right)^{2}-1\right)}{\tan \left(\sqrt{\beta}\left(\left(x-\left(8 c k^{3}+2 a k\right) t\right)\right)\right)}\right) \\
& \times e^{i\left(k x-\left(-32 a_{1}^{2} \beta^{2} f+3 c k^{4}-48 c \beta k^{2}+a k^{2}-256 c \beta^{2}-8 a \beta\right) t\right)} .
\end{aligned}
$$

When $\beta=0$, we obtain the same solution as the third solution in solution family 2 .

3.2.5. Solution Family 5

$$
\begin{aligned}
a_{0}=0, a_{1}= & \mp 2 b_{1} \sqrt{\frac{c}{b_{1}^{2} f+4 \beta^{2} c}}, b_{1}=b_{1}, a= \pm 6 f \sqrt{\frac{c}{b_{1}^{2} f+4 \beta^{2} c}} b_{1}^{2}-6 c k^{2}-20 \beta c, d=-\frac{6 \beta^{2}\left(b_{1}^{2} f+4 \beta^{2} c\right)}{b_{1}^{4}}, \\
e= & \pm \frac{4 \beta\left(\mp 2 f b_{1}^{2} \sqrt{-c / b_{1}^{2} f+4 \beta^{2} c}+15 \beta c\right)}{b_{1}^{2} \sqrt{-c / b_{1}^{2} f+4 \beta^{2} c}} \\
v= & 1 / b_{1}^{2} f+4 \beta^{2} c\left(\left( \pm 6 b_{1}^{4} f^{2} k^{2} \pm 24 b_{1}^{2} \beta^{2} c f k^{2} \mp 44 b_{1}^{4} \beta f^{2} \mp 176 b_{1}^{2} \beta^{3} c f\right) \sqrt{\frac{c}{b_{1}^{2} f+4 \beta^{2} c}}\right) \\
& \times \frac{1}{b_{1}^{2} f+4 \beta^{2} c}\left(-3 b_{1}^{2} c f k^{4}-12 \beta^{2} c^{2} k^{4}-20 b_{1}^{2} \beta c f k^{2}-80 \beta^{3} c^{2} k^{2}-2 b_{1}^{4} f^{2}+144 b_{1}^{2} \beta^{2} c f+576 \beta^{4} c^{2}\right)
\end{aligned}
$$

When $\beta<0$, we obtain

$$
q_{11,12}(x, t)=\left(\mp \frac{b_{1}\left(2 \beta \tanh \left(\sqrt{-\beta}\left(x-c_{0} t\right)\right)^{2} \sqrt{-c /\left(b_{1}^{2} f+4 \beta^{2} c\right)} \pm 1\right)}{\sqrt{-\beta} \tanh \left(\sqrt{-\beta}\left(x-c_{0} t\right)\right)}\right) e^{i(k x-v t)}
$$

where $c_{0}=8 c k^{3}+2 k\left( \pm 6 f b_{1}^{2} \sqrt{-(c) /\left(b_{1}^{2} f+4 \beta^{2} c\right)}-6 c k^{2} \quad\right.$ When $\beta>0$, we get $-20 \beta c$ ).

$$
q_{13,14}(x, t)=\left(\mp \frac{b_{1}\left(2 \beta \tan \left(\sqrt{\beta}\left(x-c_{0} t\right)\right)^{2} \sqrt{-(c) /\left(b_{1}^{2} f+4 \beta^{2} c\right)} \mp 1\right)}{\sqrt{\beta} \tan \left(\sqrt{\beta}\left(x-c_{0} t\right)\right)}\right) e^{i(k x-v t)}
$$

where $c_{0}=8 c k^{3}+2 k\left( \pm 6 f b_{1}^{2} \sqrt{-}\left((c) /\left(b_{1}^{2} f+4 \beta^{2} c\right)\right)-6 c k^{2}\right.$ $-20 \beta c$ ).

When $\beta=0$, we find

$$
q_{15,16}(x, t)=\left(-\frac{b_{1}\left(\left(x-c_{0} t\right)^{2} \mp 2 \sqrt{-\left(c / b_{1}^{2} f\right)}\right)}{\left(x-c_{0} t\right)}\right) e^{i(k x-v t)},
$$

where $c_{0}=8 c k^{3}+2 k\left( \pm 6 f b_{1}^{2} \sqrt{-\left(c / b_{1}^{2} f\right)}-6 c k^{2}\right)$.
3.3. The Exponential Rational Function Procedure. Since the balancing number is 1 , according to the exponential rational function procedure, the solution is

$$
u(\xi)=a_{0}+\frac{a_{1}}{1+e^{\xi}} .
$$

If we surrogate equation (34) into equation (13) and collect all the terms with the same order of $e^{i \xi},(i=0,1, \ldots, 5)$ together, and we make into the left-hand side of equation (4) and another polynomial in $e^{i \xi}$. Then, we 
equate each coefficient to zero to get an algebraic equation set for $a_{i}(i=0,1),\left(a_{1} \neq 0\right)$ unknown parameters as follows:

$$
\begin{aligned}
& e^{5 \xi}: d a_{0}^{5}-3 c k^{4} a_{0}-a k^{2} a_{0}+e a_{0}^{3}+v a_{0}=0 \\
& e^{4 \xi}: 5 d a_{0}^{4} a_{1}+2 f a_{0}^{2} a_{1}-a k^{2} a_{1}+v a_{1}+5 d a_{0}^{5}+c a_{1}-15 c k^{4} a_{0} \\
& +6 c k^{2} a_{1}+5 e a_{0}^{3}+3 e a_{0}^{2} a_{1}-3 c k^{4} a_{1}-5 a k^{2} a_{0}+5 v a_{0}+a a_{1}=0 \\
& e^{3 \xi}: 2 f a_{0}^{2} a_{1}+12 e a_{0}^{2} a_{1}+20 d a_{0}^{4} a_{1}+10 e a_{0}^{3}-11 c a_{1}+6 c k^{2} a_{1} \\
& +3 e a_{0} a_{1}^{2}+4 v a_{1}-+6 f a_{0} a_{1}^{2}+10 v a_{0}+10 d a_{0}^{5}+a a_{1} \\
& 4 a k^{2} a_{1}-10 a k^{2} a_{0}-30 c k^{4} a_{0}-12 c k^{4} a_{1}+10 d a_{0}^{3} a_{1}^{2}=0, \\
& e^{2 \xi}: e a_{1}^{3}-2 f a_{0}^{2} a_{1}+11 c a_{1}+18 e a_{0}^{2} a_{1}+10 e a_{0}^{3}+4 f a_{1}^{3}+10 v a_{0}-6 c k^{2} a_{1} \\
& +30 d a_{0}^{3} a_{1}^{2}-a a_{1}+9 e a_{0} a_{1}^{2}-6 a k^{2} a_{1}+10 d a_{0}^{5}-10 a k^{2} a_{0}+2 f a_{0} a_{1}^{2} \\
& +6 v a_{1}+30 d a_{0}^{4} a_{1}+10 d a_{0}^{2} a_{1}^{3}-30 c k^{4} a_{0}-18 c k^{4} a_{1}=0 \\
& e^{\xi}:-2 f a_{0}^{2} a_{1}+12 e a_{0}^{2} a_{1}+9 e a_{0} a_{1}^{2}-6 c k^{2} a_{1}-4 f a_{0} a_{1}^{2}+5 d a_{0} a_{1}^{4}-2 f a_{1}^{3} \\
& +5 e a_{0}^{3}+20 d a_{0}^{2} a_{1}^{3}-a a_{1}+20 d a_{0}^{4} a_{1}+5 v a_{0}+2 e a_{1}^{3}+30 d a_{0}^{3} a_{1}^{2} \\
& -c a_{1}-15 c k^{4} a_{0}+4 v a_{1}+5 d a_{0}^{5}-4 a k^{2} a_{1}-5 a k^{2} a_{0}-12 c k^{4} a_{1}=0, \\
& e^{0 \xi}: 3 e a_{0}^{2} a_{1}+3 e a_{0} a_{1}^{2}-a k^{2} a_{1}-3 c k^{4} a_{1}+e a_{1}^{3} \\
& +v a_{1}+d a_{1}^{5}-a k^{2} a_{0}-3 c k^{4} a_{0}+5 d a_{0}^{4} a_{1}+v a_{0} \\
& +10 d a_{0}^{3} a_{1}^{2}+10 d a_{0}^{2} a_{1}^{3}+5 d a_{0} a_{1}^{4}+e a_{0}^{3}+d a_{0}^{5}=0 .
\end{aligned}
$$

Thus, we obtain the following solutions:

Solution 1:

$$
\begin{aligned}
& d=-\frac{2 f}{a_{1}^{2}}, \\
& e=\frac{8 f}{3}, \\
& v=\frac{1}{2} a_{1}^{2} f k^{4}+\frac{5}{6} a_{1}^{2} f k^{2}-\frac{2}{3} a_{1}^{2} f .
\end{aligned}
$$$$
a=a_{1}^{2} f k^{2}+\frac{5}{6} a_{1}^{2} f,
$$$$
a_{0}=0,
$$

$$
c=-\frac{a_{1}^{2} f}{6},
$$

By substituting these values, we find

$$
q_{17}(x, t)=\frac{a_{1} e^{i\left(k x-\left((1 / 2) a_{1}^{2} f k^{4}+(5 / 6) a_{1}^{2} f k^{2}-(2 / 3) a_{1}^{2} f\right) t\right)}}{1+\cosh \left(x-\left((5 / 3) a_{1}^{2} f k+(2 / 3) a_{1}^{2} f k^{3}\right) t\right)+\sinh \left(x-\left((5 / 3) a_{1}^{2} f k+(2 / 3) a_{1}^{2} f k^{3}\right) t\right)} .
$$




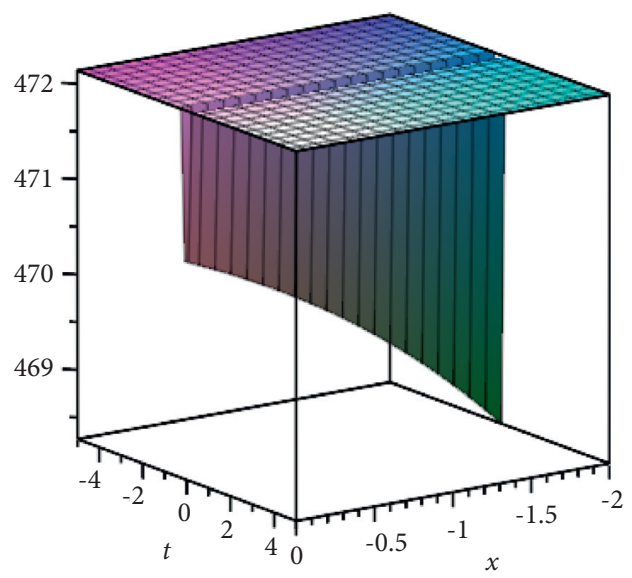

Figure 1: The dark soliton solution of $\left|q_{4}(x, t)\right|$ when $\beta=-0.05$, $c=-1, k=3, a_{1}=0.2, f=-2.5, a=-2$, within the interval $-4 \leq x$ $\leq 0,-5 \leq t \leq 5$.

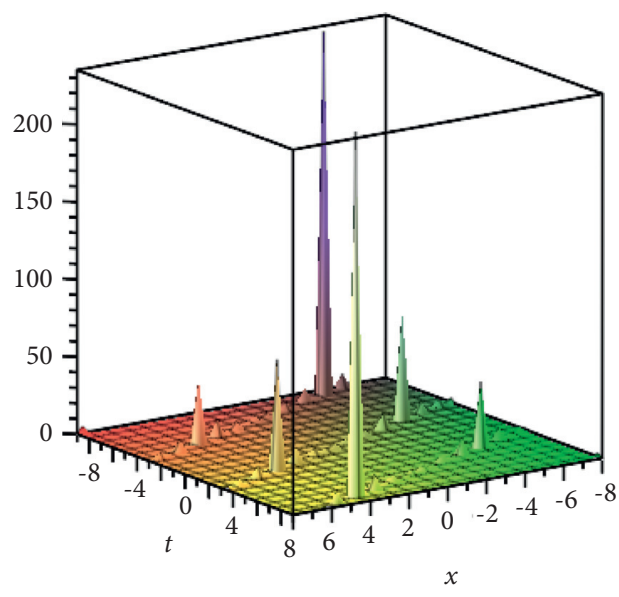

Figure 2: The periodic solution of $\left|q_{5}(x, t)\right|$ when $\beta=0.05$, $c=-1, k=3, a_{1}=0.2, f=-2.5, \quad a=-2$, within the interval $-8 \leq$ $x \leq 8,-9 \leq t \leq 9$.

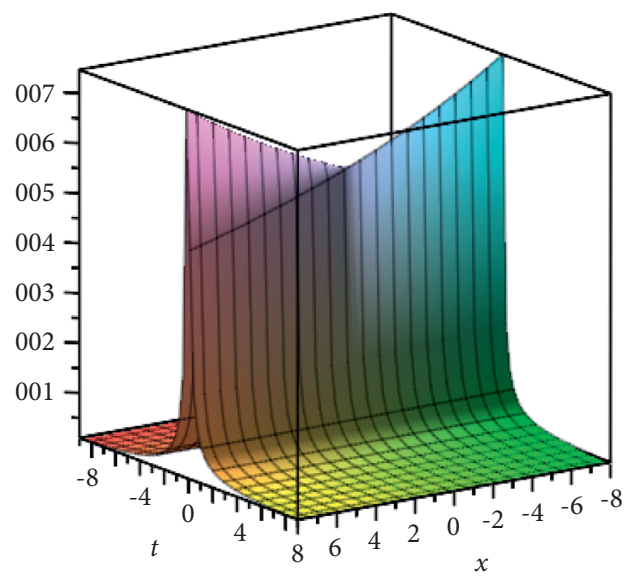

FIGURE 3: The bright soliton solution of $\left|q_{6}(x, t)\right|$ when $\beta=0$, $c=-1, k=3, a_{1}=0.2, f=-2.5, \quad a=-2$, within the interval $-8 \leq$ $x \leq 8,-9 \leq t \leq 9$.

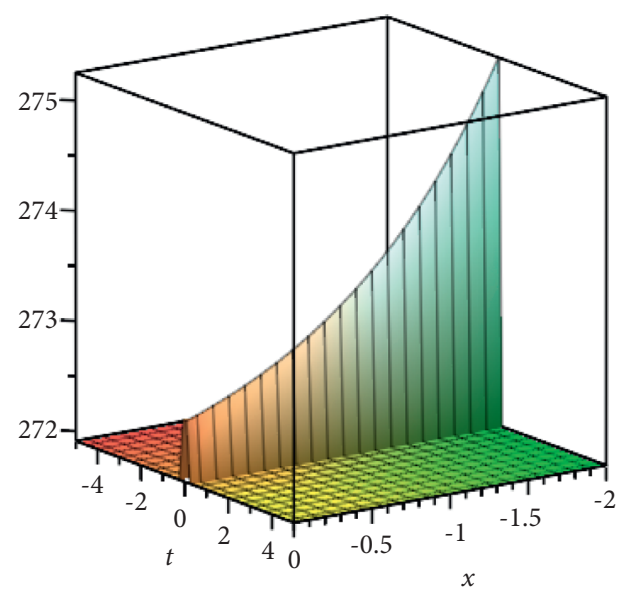

FIGURE 4: The bright soliton solution of $\left|q_{9}(x, t)\right|$ when $\beta=-0.05$, $c=-1, k=3, a_{1}=0.2, f=-2.5, a=-2$, within the interval $-2 \leq x$ $\leq 0,-5 \leq t \leq 5$.

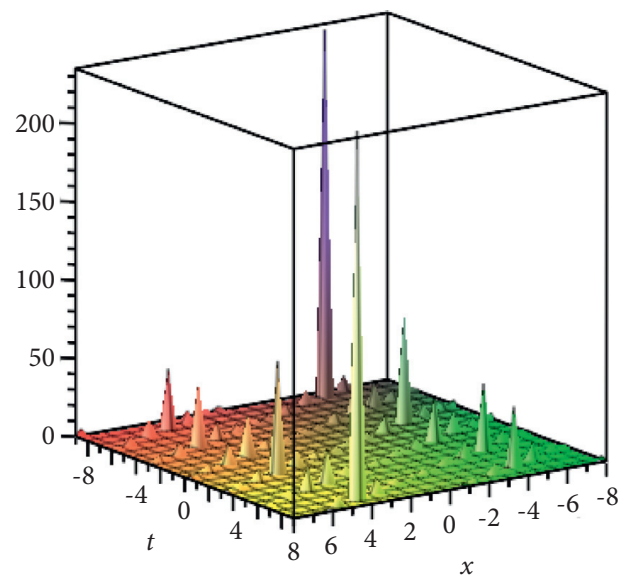

FIgURE 5: The periodic solution of $\left|q_{10}(x, t)\right|$ when $\beta=0.05, c=-1, k=3, a_{1}=0.2, f=-2.5$,

$a=-2$, within the interval $-8 \leq x \leq 8,-9 \leq t \leq 9$.

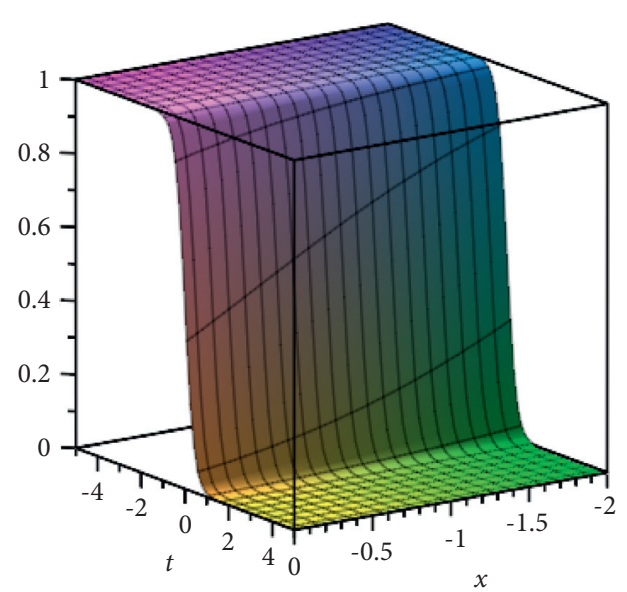

FIGURE 6: The antikink soliton solution of $\left|q_{17}(x, t)\right|$ when $a_{1}=1$, $f=2, k=-1$ within the interval $-2 \leq x \leq 0,-5 \leq t \leq 5$. 


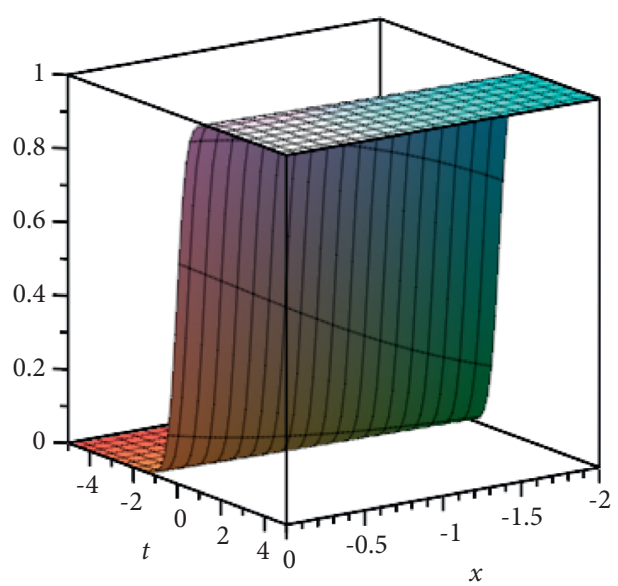

FIgURE 7: The antikink soliton solution of $\left|q_{18}(x, t)\right|$ when $a_{1}=1, f=2, k=-1$ within the interval $-2 \leq x \leq 0,-5 \leq t \leq 5$.

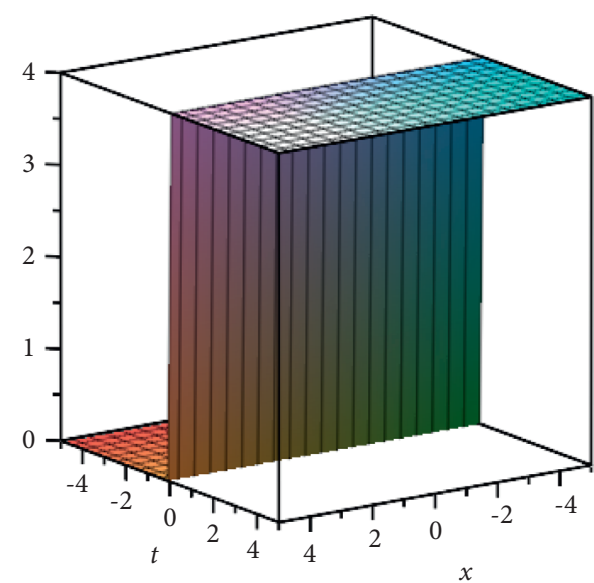

FIGURE 8: The antikink soliton solution of $\left|q_{19}(x, t)\right|$ when $a_{1}=-4, f=0.7, k=-3.5$ within the interval $-5 \leq x \leq 5,-5 \leq t \leq 5$.

Solution 2:

$$
\begin{aligned}
& a=\frac{5}{6} a_{1}^{2} f+a_{1}^{2} f k^{2}, \\
& a_{0}=-a_{1}, \\
& c=-\frac{a_{1}^{2} f}{6}, \\
& d=-\frac{2 f}{a_{1}^{2}},
\end{aligned}
$$

$$
\begin{aligned}
& e=\frac{8 f}{3}, \\
& v=\frac{1}{2} a_{1}^{2} f k^{4}+\frac{5}{6} a_{1}^{2} f k^{2}-\frac{2}{3} a_{1}^{2} f .
\end{aligned}
$$

By substituting these values, we find

$$
q_{18}(x, t)=\frac{a_{1} e^{i\left(k x-\left((1 / 2) a_{1}^{2} f k^{4}+(5 / 6) a_{1}^{2} f k^{2}-(2 / 3) a_{1}^{2} f\right) t\right)}}{1+\cosh \left(x-\left(\left(5 a_{1}^{2} f k / 3\right)+\left(2 a_{1}^{2} f k^{3} / 3\right)\right) t\right)+\sinh \left(x-\left(\left(5 a_{1}^{2} f k / 3\right)+\left(2 a_{1}^{2} f k^{3} / 3\right)\right) t\right)} .
$$


Solution 3:

$$
\begin{aligned}
a & =-\frac{e a_{1}^{2}}{2}-\frac{5 d a_{1}^{4}}{24}-\frac{a_{1}^{2} f}{4}+\frac{k^{2} d a_{1}^{4}}{4}+\frac{3 a_{1}^{2} f k^{2}}{2}, \\
a_{0} & =-\frac{a_{1}}{2} \\
c & =-\frac{d a_{1}^{4}}{24}-\frac{a_{1}^{2} f}{4},
\end{aligned}
$$

$$
\begin{aligned}
v= & -\frac{e a_{1}^{2}}{4}+\frac{k^{4} d a_{1}^{4}}{8}+\frac{3 a_{1}^{2} f k^{4}}{4}-\frac{d a_{1}^{4}}{16}-\frac{k^{2} e a_{1}^{2}}{2} \\
& -\frac{5 k^{2} d a_{1}^{4}}{24}-\frac{a_{1}^{2} f k^{2}}{4} .
\end{aligned}
$$

By substituting these values, we find

$$
\begin{aligned}
q_{19}(x, t)= & \left(-\frac{a_{1}}{2}+\frac{a_{1}}{1+\cosh \left(x-\left(a_{1}^{2} f k^{3}+k^{3} d a_{1}^{4} / 6-a_{1}^{2} f k / 2-5 k d a_{1}^{4} / 12-k e a_{1}^{2}\right) t\right)+\sinh \left(x-\left(a_{1}^{2} f k^{3}+k^{3} d a_{1}^{4} / 6-a_{1}^{2} f k / 2-5 k d a_{1}^{4} / 12-k e a_{1}^{2}\right) t\right)}\right) \\
& \times e^{i\left(k x-\left(-e a_{1}^{2} / 4+k^{4} d a_{1}^{4} / 8+3 a_{1}^{2} f k^{4} / 4-d a_{1}^{4} / 16-k^{2} e a_{1}^{2} / 2-5 k^{2} d a_{1}^{4} / 24-a_{1}^{2} f k^{2} / 4\right) t\right)} .
\end{aligned}
$$

\section{Conclusion}

NPDEs have a broad field of applications in fundamental sciences such as computer science, biology, chemistry, mathematics, physics, and engineering sciences such as chemical engineering, electricity, mechanics, and materials. The exact solutions of NPDEs have attracted considerable observation from researchers. For this reason, we have studied modified extended tanh-function and the exponential rational function procedures to determine various types of exact solutions of an integrable equation for wave packet envelope. Also, we have obtained 3D graphics of the obtained solutions (Figures 1-8). The obtained exact solutions have been verified by substituting them back into the original equation using Maple 19. According to our point of view, our obtained results have the potential to be applicable in nonlinear dynamics and optics. The obtained solutions can be classified as periodic and soliton solutions. For future works, supernonlinear, quasiperiodic, chaotic wave, and solitonic solutions will be potentially obtained. Finally, despite using Maple 19 during the calculations, the authors have noted that wxMaxima can be chosen due to the fact that is free and open-source software.

\section{Data Availability}

The datasets used or analyzed during the current study are available from the corresponding author on reasonable request.

\section{Conflicts of Interest}

The authors declare that there are no conflicts of interest regarding the publication of this paper.

\section{References}

[1] M. J. Ablowitz, "Nonlinear wave propagation," in Encyclopedia of Physics, R. Lerner and G. Trigg, Eds., AddisonWesley, Reading, MA, USA, pp. 663-664, 1981.
[2] M. T. Alquran, "Solitons and periodic solutions to nonlinear partial differential equations by the Sine-Cosine method," Appl. Math. Inf. Sci.vol. 6, no. 1, pp. 85-88, 2012.

[3] A. Akbulut, M. Kaplan, and F. Tascan, "The investigation of exact solutions of nonlinear partial differential equations by using $\exp (-\Phi(\xi))$ method," Optik, vol. 132, pp. 382-387, 2017.

[4] M. Kaplan, A. Akbulut, and A. Bekir, "Exact travelling wave solutions of the nonlinear evolution equations by auxiliary equation method," Zeitschrift für Naturforschung A, vol. 70, no. 11, pp. 969-974, 2015.

[5] K. K. Ali, H. Rezazadeh, N. Raza, and M. Inc, "Highly dispersive optical soliton perturbation with cubic-quintic-septic law via two methods," International Journal of Modern Physics B, vol. 35, no. 27, Article ID 2150276, 2021.

[6] S. W. Yao, L. Akinyemi, M. Mirzazadeh, M. Inc, K. Hosseini, and M. Senol, "Dynamics of optical solitons in higher-order Sasa-Satsuma equation," Results in Physics, vol. 30, no. 3, Article ID 104825, 2021.

[7] H. Durur, A. Kurt, and O. Tasbozan, "New travelling wave solutions for KdV6 equation using sub equation method," Applied Mathematics and Nonlinear Sciences, vol. 5, no. 1, pp. $455-460,2020$.

[8] M. Mirzazadeh, L. Akinyemi, M. Senol, and K. Hosseini, “A variety of solitons to the sixth-order dispersive $(3+1)$ -dimensional nonlinear time-fractional Schrödinger equation with cubic-quintic-septic nonlinearities," Optik, vol. 241, no. 8, Article ID 166318, 2021.

[9] W.-X. Ma, M. S. Osman, S. Arshed, N. Raza, and H. M. Srivastava, "Practical analytical approaches for finding novel optical solitons in the single-mode fibers," Chinese Journal of Physics, vol. 72, pp. 475-486, 2021.

[10] M. S. Osman, "Multi-soliton rational solutions for quantum Zakharov-Kuznetsov equation in quantum magnetoplasmas," Waves in Random and Complex Media, vol. 26, no. 4, pp. 434-443, 2016.

[11] M. Inc, H. Rezazadeh, H. Rezazadeh et al., "New solitary wave solutions for the conformable Klein-Gordon equation with quantic nonlinearity," Aims Mathematics, vol. 5, no. 6, pp. 6972-6984, 2020.

[12] T. D. Leta, W. Liu, A. El Achab, H. Rezazadeh, and A. Bekir, "Dynamical behavior of traveling wave solutions for a $(2+1)$ dimensional Bogoyavlenskii coupled system," Qualitative Theory of Dynamical Systems, vol. 20, no. 1, pp. 1-22, 2021. 
[13] H. Rezazadeh, M. Younis, M. Shafqat-Ur-Rehman, M. Eslami, M. Bilal, and U. Younas, "New exact traveling wave solutions to the (2+1)-dimensional Chiral nonlinear Schrödinger equation," Mathematical Modelling of Natural Phenomena, vol. 16, p. 38, 2021.

[14] A. Biswas, Y. Yildirim, E. Yasar, Q. Zhou, S. P. Moshokoa, and M. Belic, "Optical solitons for Lakshmanan-Porsezian-Daniel model by modified simple equation method," Optik, vol. 160, pp. 24-32, 2018.

[15] J. Manafian, "Optical soliton solutions for Schrödinger type nonlinear evolution equations by the $\tan (\Phi(\xi) / 2)$-expansion method," Optik, vol. 127, no. 10, pp. 4222-4245, 2016.

[16] N. A. Kudryashov, "Method for finding highly dispersive optical solitons of nonlinear differential equations," Optik, vol. 206, p. 163550, 2020.

[17] G. Fibich, The Nonlinear Schrödinger Equation Singular Solutions and Optical Collapse, Springer, Berlin, Germany, 2015.

[18] E. M. E. Zayed and K. A. E. Alurrfi, "Extended auxiliary equation method and its applications for finding the exact solutions for a class of nonlinear Schrödinger-type equations," Applied Mathematics and Computation, vol. 289, pp. 111-131, 2016.

[19] E. M. E. Zayed and A.-G. Al-Nowehy, "Solitons and other exact solutions for a class of nonlinear Schrödinger-type equations," Optik, vol. 130, pp. 1295-1311, 2017.

[20] V. G. Kartavenko, "Soliton-like solutions in nuclear hydrodynamics," Soviet Journal of Nuclear Physics, vol. 40, pp. 240-246, 1984.

[21] A. Kumar, S. N. Sarkar, and A. K. Ghatak, "Effect of fifthorder nonlinearity in refractive index on Gaussian pulse propagation in lossy optical fibers," Optics Letters, vol. 11, no. 5, pp. 321-323, 1986.

[22] I. V. Barashenkov and V. G. Makhankov, "Soliton-like "bubbles" in a system of interacting bosons," Physics Letters A, vol. 128 , no. $1-2$, pp. $52-56,1988$.

[23] N. A. Kudryashov, "Optical solitons of model with integrable equation for wave packet envelope," Chaos, Solitons \& Fractals, vol. 141, p. 110325, 2020.

[24] W. Hereman and A. Nuseir, "Symbolic methods to construct exact solutions of nonlinear partial differential equations," Mathematics and Computers in Simulation, vol. 43, no. 1, pp. 13-27, 1997.

[25] S. A. Elwakil, S. K. El-labany, M. A. Zahran, and R. Sabry, "Modified extended tanh-function method for solving nonlinear partial differential equations," Physics Letters A, vol. 299, no. 2-3, pp. 179-188, 2002.

[26] B. Ghanbari and M. Inc, "A new generalized exponential rational function method to find exact special solutions for the resonance nonlinear Schrödinger equation," The European Physical Journal Plus, vol. 133, no. 4, p. 142, 2018.

[27] M. Kaplan, A. R. Butt, H. Thabet, A. Akbulut, N. Raza, and D. Kumar, "An effective computational approach and sensitivity analysis to pseudo-parabolic-type equations," Waves in Random and Complex Media, pp. 1-15, 2021. 